\title{
Blunt Trauma Chest: A Study on Clinical Pattern
}

\author{
Dr B Anil Kumar ${ }^{1}$, Dr G Raghuveer Chakravarthy ${ }^{2,}$ Dr A Bharath ${ }^{3}$ \\ ${ }^{1}$ Dr B Anil Kumar, Professor, General Surgery, Dr.Pinnamaneni Siddartha Institute Of Medical Sciences \& \\ Research Foundation, Chinaoutapalli, Krishna Dist, Andhra Pradesh, India. \\ ${ }^{2}$ Dr G Raghuveer Chakravarthy, Assistant Professor, General Surgery, Dr.Pinnamaneni Siddartha Institute Of \\ Medical Sciences \& Research Foundation, Chinaoutapalli, Krishna Dist, Andhra Pradesh, India. \\ ${ }^{3}$ Dr A Bharath ,Junior Resident, General Surgery, Dr.Pinnamaneni Siddhartha Institute Of Medical Sciences \& \\ Research Foundation, Chinaoutapalli, Krishna Dist, Andhra Pradesh, India.
}

\begin{abstract}
Background: Chest injuries constitute a continuing challenge to the trauma or general surgeon practicing in a developing country. This study reviews the pattern and management of these injuries mainly by general surgeons.

Methods: A cross sectional study of 200 patients attending to emergency room in Tertiary care teaching Hospital with chest injuries between January 2013 and December 2016.

Results: Over 3 years period, 200 patients with various forms of chest injuries were managed among them Males were commonly affected than female with 4.5:1 ratio, Maximum incidence in age group 41-60 years with $66 \%$. Mean age 44.78 years, Mean Hospital stay 10.78 days

Conclusion: Most of the injuries are due to Motor Vehicle Accidents (MVA), Commonest injury being Rib Fracture (78\%) followed by Hemothorax (60\%) and Pneumothorax (44\%) commonest associated injury is Clavicle Fracture. Most commonest solid abdominal organ injury is Liver (16\%) followed by Spleen (8\%). Overall $76 \%$ patients required tube thoracostomy and $24 \%$ patients were managed conservatively. Overall Mortality was $6 \%$ in the study.
\end{abstract}

Keywords: Chest trauma, Pattern, MVA, Thoracostomy

\section{Introduction}

Injuries to the chest are common, with up to $20 \%$ of trauma patients presenting with thoracic injuries. Chest trauma is a significant source of morbidity and mortality. The incidence of chest injuries are on a rise, with motor vehicle accidents (MVA) playing the most important role. It is estimated that by the year 2020, 8.4 million people will die every year from injury, and injuries from road traffic accidents will be the third most common cause of disability worldwide and the second most common cause in the developing world [1]. According to the National Trauma Database (NTDB), chest injuries are present in $13.8 \%$ of all blunt trauma patients. The overall mortality rate of chest trauma is $8.4 \%$. The mortality rate of blunt thoracic trauma ranges from $9.5 \%-47.5 \%$, depending upon the severity of injury sustained. The most common cause is MVA, followed by fall. MVAs account for 70-80\% of such injuries. Industrial crush injuries, sports, crime and blast injuries are other causes. Direct compression, as well as deceleration and rotational physical mechanisms contribute to blunt thoracic trauma.Blunt injury to the chest can affect any one or all components of the chest wall and thoracic cavity. These components include the bony skeleton (ribs, clavicles, scapulae, sternum), lungs and pleurae, trachea bronchial tree, oesophagus, heart, great vessels of the chest, and the diaphragm. Often a combination of injuries is present.

Blunt thoracic trauma is often associated with injuries to other organs, especially the abdominal solid organs as the upper part of the abdomen is overlapped by the thoracic cage. Optimal care of severely injured patients requires a coordinated approach from the point of injury, through a hospital facility organised to cope with the demands of looking after multisystem problems, to a rehabilitation structure that can return the patient to his or her maximum potential level of function within society. Although sophisticated prehospital and trauma centre systems have been shown to reduce the number of preventable deaths after trauma [2], maximum impact in reducing the burden of trauma must come from injury prevention strategies.

\section{Objectives}

1) The proportionate various types of injuries.

2) The various mechanisms by which chest injuries are sustained.

3) The proportion of associated abdominal solid organ injuries. 


\section{Materials And Methods}

Study design: Cross-sectional study

Duration of study: 3 years

Number of subjects: 200

Setting: Tertiary Care Teaching Hospital

Inclusion criteria:

Patients presenting to emergency department with blunt trauma to chest

Exclusion criteria:

Patients with severe head injury

\section{Results}

The study is conducted in patients presenting to our emergency department with blunt trauma to chest. The mechanism of injury, time of injury, clinical assessment will be accurately documented. Thereafter the patient will be subjected to the appropriate primary investigations viz Haemoglobin concentration, PCV \& chest $\mathrm{X}$-ray. Higher radiological investigations viz ultra sonogram and CT thorax will be done as and when required. The patients will be duly followed up during their hospital stay to monitor their progress and to identify the development of complications. Maximum number of patients belonged to the age group 41 to 60 years, i.e $66 \%$. Mean age is 44.78 years.

\begin{tabular}{|c|c|}
\hline Age (years) & Number of patients \\
\hline $13-20$ & 8 \\
\hline $21-30$ & 44 \\
\hline $31-40$ & 28 \\
\hline $41-50$ & 44 \\
\hline $51-60$ & 48 \\
\hline $61-70$ & 28 \\
\hline$>70$ & nil \\
\hline
\end{tabular}

$82 \%$ of the patients were males. We had only 36 female cases, i.e a male to female ratio of $4.5: 1$

\begin{tabular}{|c|c|}
\hline Sex & number of patients \\
\hline Male & 164 \\
\hline Female & 36 \\
\hline
\end{tabular}

Average hospital stay 10.78 days

\section{Types of chest injuries}

The commonest injury in this series is rib fracture (78\%) followed by haemothorax $(60 \%)$ and pneumothorax (44\%). Often a combination of these injuries were present. Rib fractures were often multiple $(58 \%)$. The least common injuries were lung laceration, cardiac and great vessel injuries. We had 12 cases of flail chest. Lung contusion (32\%) was the most common parenchymal lung injury. Lung collapse was seen in $12 \%$ of cases and we had 4 cases of lung laceration. We had 4 cases each of cardiac, aortic and diaphragmatic injuries.

\section{Type of injury}

Rib fracture

Number
156
8
116
20
12
120
88
40
64
24
4
12
4
4

Graph 1 : Types of chest injuries 


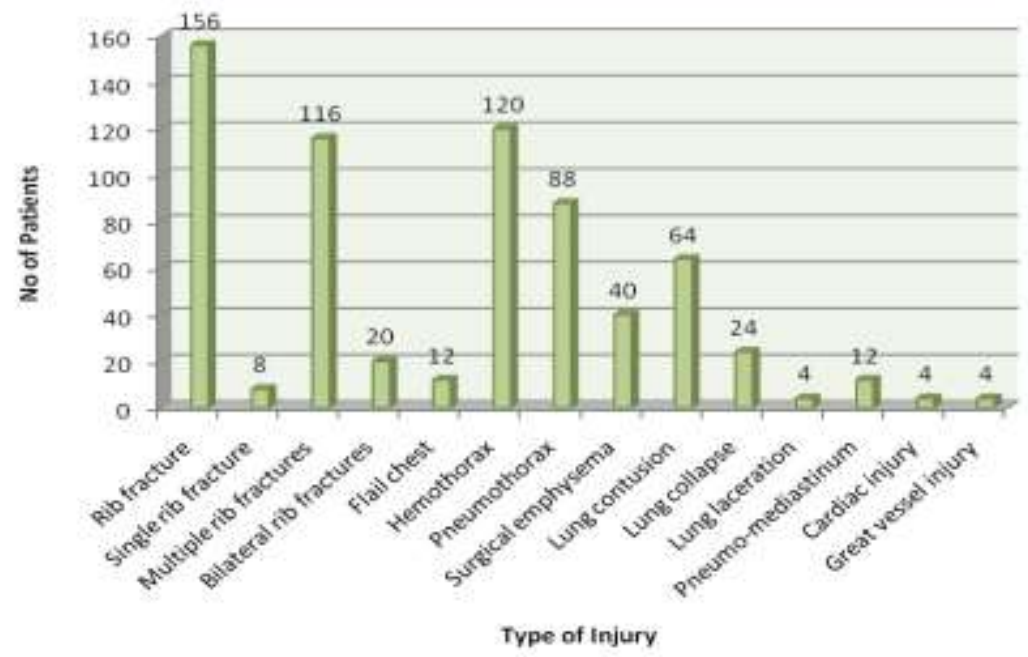

\section{Associated injuries}

The most common associated injury was clavicle fracture (22\%). Vertebral injuries were seen in $12 \%$ of cases.The most common associated abdominal solid organ injury was that of fiver (16\%), followed by spleen $(8 \%)$, pancreas $(2 \%)$ and kidney $(2 \%)$. Hemoperitoneum was found radiologically in $24 \%$ of cases.

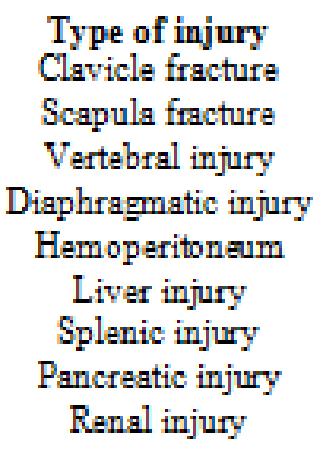

Number
44
12
24
4
48
32
16
4
4

Graph 2 : Associated injuries

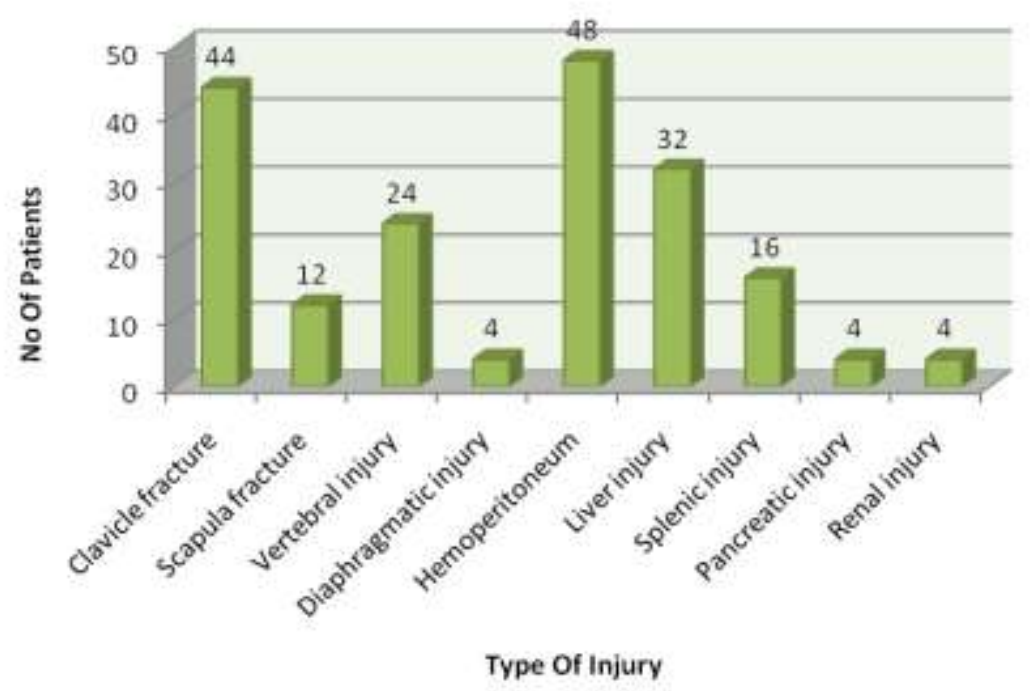

\section{Causes of injuries}

Majority of patients sustained injury following MVA or fall from height. MVAs contributed 50\% and fall from height $38 \%$. We had 3 patients who sustained chest trauma following fall of heavy objects onto chest. 
Two patients sustained injuries following railway accidents. One patient sustained injury due to fall following attack by Stray dog.

\section{Cause of injury}

Motor vehicle accidents

Fall from height

Fall of objects onto chest

Railway accidents

Animal attack

Number
100
76
12
8
4

Graph 3 : Causes of injury

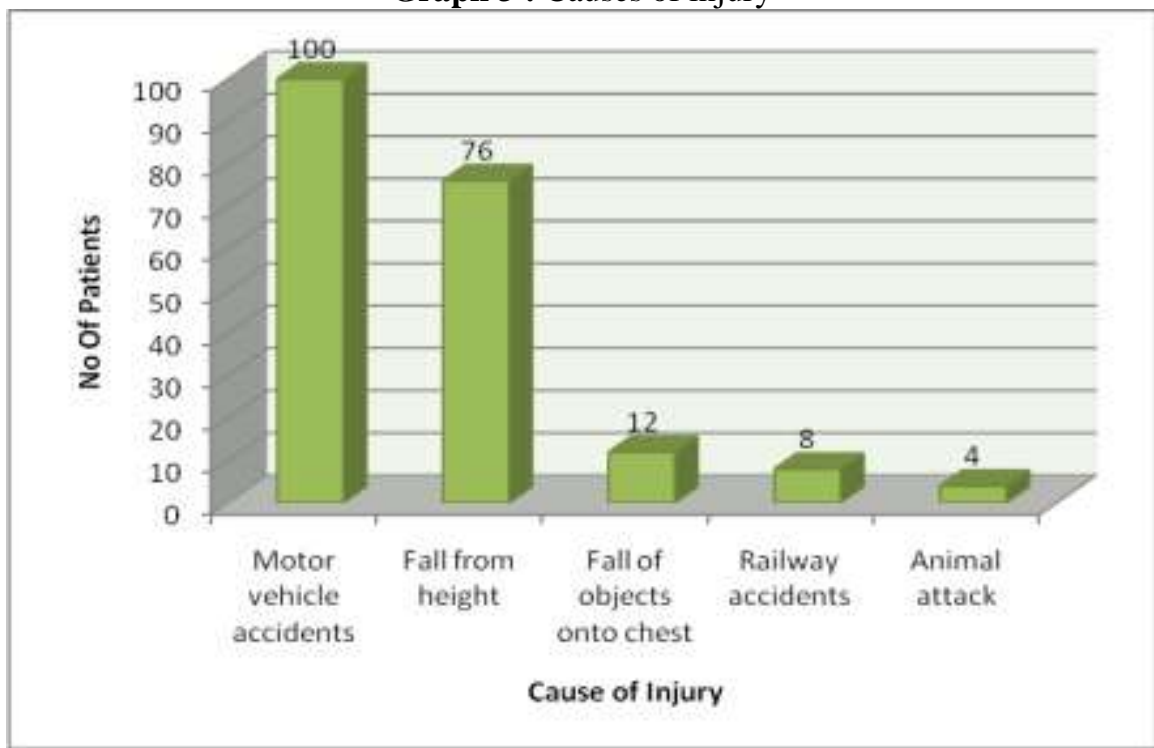

\section{Mechanism of iniury}

The three major mechanisms involved in blunt thoracic trauma are direct compression, rotational mechanism and deceleration. Direct compression played sole role in $96 \%$ of cases. Rotational mechanism and deceleration played role in 16 and 12 cases respectively.

$\begin{array}{ll}\text { Mechanism of injury } & \text { Number } \\ \text { Direct compression } & 200 \\ \text { Rotational mechanism } & 16 \\ \text { Deceleration } & 12\end{array}$

\section{Modalities of Management}

Altogether $76 \%$ cases required tube thoracotomy. $22 \%$ cases required bilateral tube thoracostomy. Only 4 cases of cardiac injury required thoracotomy. 8 patients underwent laparotomy. $24 \%$ of cases were managed conservatively.

\section{Modalities of management}

Unilateral ICD insertion

\section{Number}

108

Bilateral ICD insertion

44

Mechanical ventilation

24

Tracheostomy

12

Thoracotomy

Laparotomy

8

Conservative 


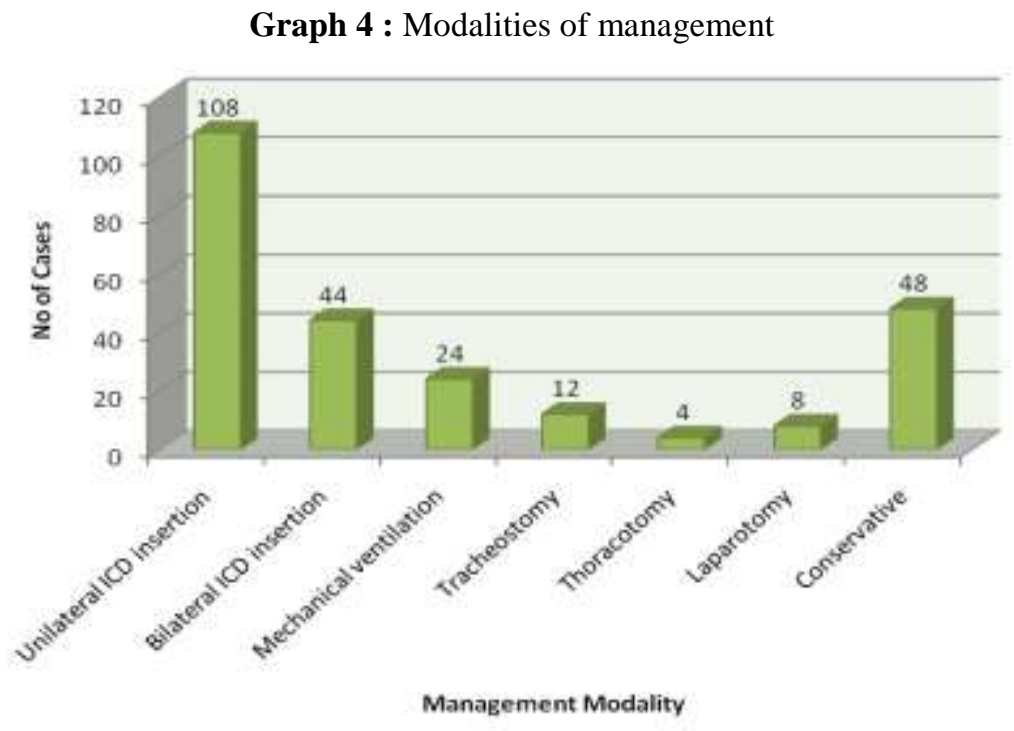

\section{Complications}

Pneumonia was the most common complication encountered followed by septicaemia and pyothorax.

\section{Complications}

Pneumonia-consolidation

Septicaemia

Number

Pyothorax

20

COPD exacerbation

MODS

ARF

Mortality

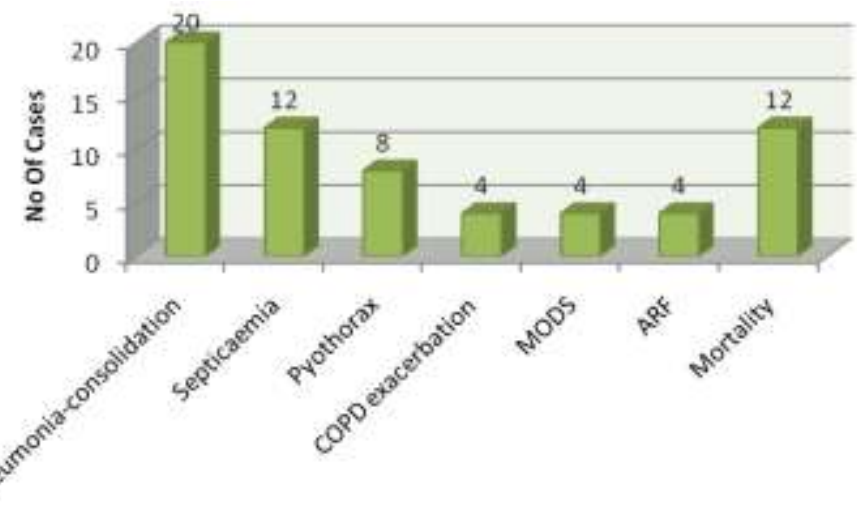

Complications

Overall mortality rate in this series is $6 \%$.

\section{Discussion}

The American Academy of Science describes trauma as the "neglected disease of modern society"[3]. Trauma is usually considered as the main cause of mortality and morbidity in individuals between 1 - 44 years [4].Chest injuries are common reasons for emergency medical care. In civilian practice, chest trauma is commonly caused by road traffic accidents. Most of the injuries are found among young adult males between the ages of 20 and 40 years the very productive period of life. [5-7].

A total of 200 patients with blunt thoracic trauma were studied in this series, Majority of patients sustained injury following MVA or fall from height. MVAs contributed $50 \%$ and fall from height $38 \%$. We had 12 patients who sustained chest trauma following fall of heavy objects onto chest. Two patients sustained injuries following railway accidents, 4 patients sustained injuries due to fall following attack by stray dog. 
World over MVA is the most common cause of blunt chest trauma. In a study conducted by Robert.M.Shorr etal of Maryland Institute for Emergency Medical Services Systems (MIEMSS), MVAs contributed $70.9 \%$ of cases, falls $7.6 \% \%$ and animal attack $0.2 \%$ (8). In my series, MVA, falls and animal attack contributed 50\%, 38\% and 2\% respectively. Fall of objects onto chest while carrying load contributed $6 \%$ of cases. This reflects that there is considerable difference in the modes of injury between developed and developing nations. Here fall from height contributed substantially. Most of the cases were occupational hazards especially fall from coconut tree and arecanut tree, and fall sustained by masons at construction sites. This reflects the difference in occupational patterns of these nations. In a study - "Pattern and outcome of chest injuries at Bugando Medical Centre in Northwestern Tanzania" done by Lema M.K et al, MVAs contributed $50.7 \%$ of cases. In our region animal attack and railway accidents are more common. More number of railway accidents may be attributed to lack of safety precautions and unmanned level crosses. Maximum number of patients belonged to the age group 41 to 60 years, i.e., $66 \%$. Mean age is 44.78 years. In the MIEMSS study the mean age was 36.9 years. $82 \%$ of the patients were males. We had only 9 female cases, i.e., a male to female ratio of $4.5: 1$. In the MIEMSS study, the ratio is $2.7: 1$. In a study done in Qatar, " Rib fracture patterns predict thoracic chest wall and abdominal solid organ injury" by Al Hassani A et al, the sex ratio was $21 \%$ (9). This shows a considerable difference in the sex ratio between various nations. This can be attributed mainly to the cultural and social background of these nations. In developed nations, more ladies sustain injuries mainly because more ladies drive motor vehicles compared to developing nations. Mean hospital stay in this study was 10.78 days. It was 15 days in a study conducted in the Department of Thoracic and Cardiovascular Surgery Erciyes University Medical Faculty Kayseri, Turkey, by Cemal Kahraman et al "Blunt Thoracic Trauma: Analysis of 1730 Patients" (10). Mean hospital stay in a study conducted by Cardiothoracic Surgery Unit Lagos University Teaching Hospital College of Medicine of University of Lagos, Lagos, Nigeria by Martins Oluwafemi Thomas et al was 6.6 days (11). The commonest injury was rib fracture with or without associated pneumothorax, hemothorax, surgical emphysema, lung contusion and so on. Often a combination of these injuries were present. Incidence of rib fracture was $78 \%$, compared to $62 \%$ in the study conducted in Nigeria. Pure rib fractures were treated conservatively. 24 patients required intercostal nerve block for 3-4 days. We had 12 cases of flail chest, i.e., $6 \%$, compared to $5.5 \%$ in the Nigerian study.

All three cases required tube thoracostomy. Four cases required mechanical ventilation and tracheostomy. Incidence of pneumothorax was $44 \%$ as compared to $24 \%$ in the Nigerian study and $18.45 \%$ in the MIEMSS study. All cases of pneumothorax were treated with tube thoracostomy. ICD was removed usually within 5 to 6 days. Twelve patients had pneumo-mediastinum. Incidence of hemothorax was $60 \%$ as compared to $20 \%$ in the Nigerian study. Minimal hemothorax found on CT were treated conservatively. Most cases required ICD. CT was found to be more sensitive for hemothorax, rib fractures and parenchymal lung injuries. Prophylactic antibiotics, bronchodilators, nebulisation and incentive spirometry were given to patients who underwent tube thoracostomy. Decision for ICD was most often taken based on Plain chest $\mathrm{x}$-ray findings. Chest $\mathrm{x}$-ray (CXR) was found to be sensitive particularly for rib fractures, pneumothorax and surgical emphysema. CXR showed mediastinal widening in the only case of thoracic aortic injury in this series. ICD tube was inserted in the triangle of safety in all cases. All cases of lung collapse were partial and most improved with incentive spirometric exercise. One case had residual collapse during follow-up. We had 4 cases of lung laceration and it required thoracostomy only. We had 4 cases of diaphragmatic injury. Left dome was disrupted with herniation of splenic flexure and gastric fundus into thoracic cavity in 2 cases underwent laparotomy and repair of diaphragm. We had 4 cases of cardiac injury i.e., a $2 \%$ incidence as compared to $0.8 \%$ in the study conducted in Nigeria. One patient sustained ventricular septal rupture following fall from bicycle. Emergency closure of the defect with PTFE was done under cardiopulmonary bypass. On follow-up he had mild residual defect with pan systolic murmur. One patient sustained two tears in ascending aorta with surrounding hematoma formation (post-mortem report) . He died inspite of resuscitative efforts.

Altogether we had 18 cases of associated fractures, i.e $36 \%$ as compared to $22.4 \%$ in the study conducted in Turkey. The most common associated fracture with blunt chest trauma was that of clavicle(44 cases, i.e., 22\%),12\% cases had vertebral fractures. $6 \%$ cases had scapula fracture. $22 \%$ cases sustained abdominal solid organ injury. In a study conducted by Section of Trauma Surgery, Department of Surgery, Hamad General Hospital Doha, Qatar, the proportion of abdominal solid organ injury was $29 \%$. They found liver injuries in $13.5 \%$ cases as opposed to $16 \%$ in our series. Splenic trauma was found in $15 \%$ and $8 \%$ cases respectively in their and our study. Liver injury was the most common abdominal solid organ injury followed by spleen. 32 patients had liver injury. Four cases required laparotomy and suturing of liver laceration. All other cases improved with conservative measures. Sixteen patients sustained splenic injury. All were managed conservatively. We had four cases each of pancreatic and renal injury. The three major mechanisms involved in blunt thoracic trauma are direct compression, rotational mechanism and deceleration. Direct compression played sole role in $96 \%$ of cases. Rotational mechanism and deceleration played role in 16 and 12 cases respectively. Altogether 152 cases required tube thoracostomy, 44 underwent bilateral procedure. i.e., $76 \%$ as compared to 
$82.4 \%$ in the Nigerian study. The tube was inserted in the triangle of safety in supine position in all cases. They were given antibiotics throughout the period of intercostal drainage. The decision for tube removal was taken based on clinical \& radiological improvement as well as the amount of drainage and absence of air leak. We had four cases of thoracotomy as compared to 67 out of 896 patients in the study conducted in Nigeria. 48 cases were managed conservatively, i.e., $24 \%$ as compared to $9.8 \%$ in the Nigerian study. Mechanical ventilation was given for 24 patients. Most of them were given SIMV for a few days. 8 patients underwent laparotomy. Four under patients underwent suturing of liver laceration and the other repair of diaphragm. Most common complication seen was pneumonia (10\%), All cases responded to antibiotics and supportive therapy. In the study conducted in Turkey, the most common causes of morbidity were atelectasis $(10.06 \%)$ and pneumonia $(5.32 \%)$. We had a total of Twelve deaths, i.e., $6 \%$ mortality rate as compared to $5.61 \%$ in the Turkish study and $15.5 \%$ in the MIEMSS study.

\section{Conclusion}

1) Motor vehicle accidents were the leading cause of blunt chest trauma in this series, followed by fall from height. Direct compression of chest against static surfaces or objects contributed to almost all cases, with rotational mechanism and deceleration playing minor roles.

2) Males were more often affected with a sex ratio of $4.5: 1$. Maximum number of patients belonged to the age group 41 to 60 years, i.e., $66 \%$. Mean age is 44.78 years. The mean hospital stay was 10.78 days.

3) The commonest injury was rib fracture (78\%) followed by hemothorax $(60 \%)$ and pneumothorax (44\%).Often a combination of these injuries were present. Lung contusion $(32 \%)$ was the most common parenchymal lung injury. Lung collapse was seen in $12 \%$ of cases and we had one case of lung laceration. We had Four cases each of cardiac, aortic and diaphragmatic injuries.

4) The most common associated injury was clavicle fracture (22\%). The most common abdominal solid organ injury was that of liver (16\%), followed by spleen ( $8 \%)$, pancreas $(2 \%)$ and kidney (2\%).

5) Altogether $76 \%$ cases required tube thoracostomy of which $22 \%$ cases required a bilateral procedure. Four cases of cardiac injury required thoracotomy. 8 patients underwent laparotomy. $24 \%$ of cases were managed conservatively. Pneumonia was the most common complication encountered followed by septicaemia and pyothorax. The overall mortality rate in this series was $6 \%$.

\section{References}

[1]. Murray CJ, Lopez AD. Alternative projections of mortality and disability by cause 1990-2020: Global Burden of Disease Study. Lancet 1997; 349(9064): 1498-504.

[2]. West JG, Trunkey DD, Lim RC. Systems of trauma care: a study in two countries. Arch Surg 1979; 114: $455-60$.

[3]. J.M.Ryan,Accident and Emergency,in RCG Russel,Williams NS, Bulstrode LJK(Eds).Bailey and Love's Short Practice of Surgery23rdEdn(London:Arnold Publishers,2000)270-280

[4]. Baradaran H, Salimi J, Nassaji-Zavareh M, Khaji A, Rabbani A. Epidemiological study of patients with penetrating abdominal trauma in Tehran-Iran. Acta Medica Iranica.2007;45(4):305-8.

[5]. Inci I, Ozçelik I, Tacyildiz O, Nizam N, Eren G, Ozen DB. Penetrating chest injuries: unusually high incidence of high velocity gunshot wounds in civilian practice. World J Surg 1998; 22: 438 - 442.

[6]. Cooper C, Militello P. The multiple injured patient: Maryland shock trauma protocol approach. Sermin Thoracic Cardiovasc Surg 1992; $4: 163$.

[7]. Vasquez TC, Castaneda E, Bazan N. Management of 240 cases of penetrating thoracic injuries. Injury 1997; $28: 45$ - 49.

[8]. Robert M. Shorr,et al. Blunt Thoracic Trauma-Analysis of 515 Patients, Ann surg. 1987 ; 206(2):200-205.

[9]. A1-Hassani A et al. Rib fracture patterns predict thoracic chest wall and abdominal solid organ injury, The American Surgeon 2010 ;76(8):888- 91

[10]. Cemal Kahraman et al. Asian Cardiovasc ThoracAnn 1998;6:308-312

[11]. Oluwafemi Thomas, Ezekiel O Ogunleye. Asian Cardiovasc Thorac, Ann 2009; 17:608-611 